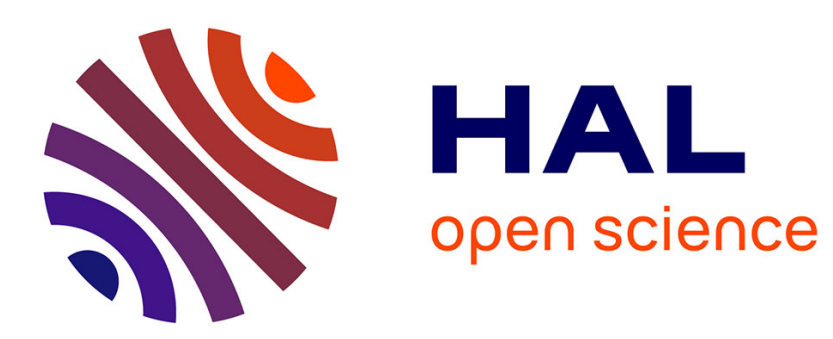

\title{
Analytical model of a longitudinal hollow cathode discharge
}

G J M Hagelaar, D B Mihailova, J van Dijk

\section{To cite this version:}

G J M Hagelaar, D B Mihailova, J van Dijk. Analytical model of a longitudinal hollow cathode discharge. Journal of Physics D: Applied Physics, 2010, 43 (46), pp.465204. 10.1088/0022$3727 / 43 / 46 / 465204$. hal-00569748

\section{HAL Id: hal-00569748 \\ https://hal.science/hal-00569748}

Submitted on 25 Feb 2011

HAL is a multi-disciplinary open access archive for the deposit and dissemination of scientific research documents, whether they are published or not. The documents may come from teaching and research institutions in France or abroad, or from public or private research centers.
L'archive ouverte pluridisciplinaire HAL, est destinée au dépôt et à la diffusion de documents scientifiques de niveau recherche, publiés ou non, émanant des établissements d'enseignement et de recherche français ou étrangers, des laboratoires publics ou privés. 
Analytical model of a longitudinal hollow cathode discharge

G. J. M. Hagelaar ${ }^{1,2,4}$, D. B. Mihailova ${ }^{3}$, and J. van Dijk ${ }^{3}$

1 Université de Toulouse; UPS, INP; LAPLACE (Laboratoire Plasma et Conversion d'Energie); 118 route de Narbonne, F-31062 Toulouse Cedex 9, France

${ }^{2}$ CNRS; LAPLACE; F-31062 Toulouse, France

${ }^{3}$ Department of Applied Physics, Eindhoven Université of Technology, P.O. Box 513, 5600 MB Eindhoven, The Netherlands

${ }^{4}$ E-mail: gerjan.hagelaar@laplace.univ-tlse.fr

PACS: 52.80.-s, 52.50.Nr, 52.25.Jm, 51.50.+v

Index terms: Hollow cathode discharge, analytical modelling, self-sustainment condition, hollow-cathode effect

Abstract

This paper presents a simple analytical model of a longitudinal hollow cathode discharge used in metal vapor lasers. The model describes the principle relations between the voltage, current, plasma density, and the axial structure of the discharge. Contrary to standard DC discharges, this discharge does not require electron multiplication in the cathode fall to produce ions, but rather to satisfy the electron energy balance. A self-sustainment condition is obtained from the energy balance per electron-ion pair. From this, it follows that there is a maximum voltage at which the cathode fall thickness tends to zero and the current density tends asymptotically to infinity. The discharge develops axial non-uniformity and an axial electric field in order to evacuate the created electrons to the anode, such that the characteristic time for transport losses is the same for electrons as for ions. The axial profiles of the current density, plasma density, and potential are obtained from the electron continuity equation. It is shown that additional energy absorption from the axial field, similar to electron heating in DC positive columns, modifies the self-sustainment condition and thus leads to a shift in the voltage-current characteristic, depending on the cathode length. 


\section{Introduction}

Hollow cathode discharges (HCDs) are widely used in applications in different fields: laser technology, atomic spectroscopy, UV generators, vacuum microelectronics, materials processing, etc. Although these discharges feature a large variety of configurations, they are generally characterized by the so-called hollow cathode effect: an exceptionally high discharge current, compared to conventional DC discharges at the same voltage, due to the cathode surface surrounding (a large part of) the plasma. The hollow cathode effect is accompanied by exceptionally high plasma density, intensive light emission, and cathode sputtering, properties of great interest for the applications.

The general physical principles of HCDs have been studied for many decades and are discussed in many papers on the basis of experiments, analytical models, and numerical simulations. [1-14] Several phenomena are considered responsible for the hollow cathode effect, in particular the electrostatic trapping of fast electrons in an oscillating motion inside the cathode, known as the pendulum effect [4,6], and the enhanced secondary-electron emission by UV photons [1] and ions [9] created in the negative glow plasma inside the cathode. A number of papers is devoted to the role of metal vapor atoms due to cathode sputtering. $[2,10]$ However, the importance of each of these phenomena depends on the HCD geometry. [9]

In this paper we present a simple analytical model of HCDs that are used in metal vapor lasers, both as a metal vapor source by cathode sputtering and as an active medium to excite the laser transition of the metal atoms or ions. [15] These HCDs have an elongated cylindrical cathode geometry, coinciding with the laser cavity. Different configurations are used for the anode. In the so-called transversal configuration, the anode is positioned beyond a narrow slit in the cathode cylinder all along its length. This paper, however, focuses on the longitudinal configuration, where the anode is a ring at the end of the cathode cylinder and the discharge involves axial electron transport.

The longitudinal configuration has a more stable discharge operation but leads to axial nonuniformity of the discharge. Experimentally, it was observed [11,16-18] that (1) the current density and optical emission decrease along the cathode cylinder axis as a function of distance 
from the anode; (2) the voltage-current characteristic is very flat but shifted in voltage when the cathode length is changed; (3) these features are rather independent of pressure, gas composition, and metal vapor. In several previous publications [16-18], we reproduced these experimental findings by comprehensive two-dimensional numerical simulations. The analytical model presented here aims at interpreting our previous results. We revisit and combine some elementary theories and extend them to account for the axial non-uniformity of the longitudinal HCD. In view of our earlier findings, we focus on the main discharge properties such as current density, plasma density, and potential distribution, rather than plasma chemistry and metal vapor dynamics. The next section gives a more detailed introduction to the issues addressed in this paper.

2. Discharge configuration and physical principles considered in this paper

The geometrical configuration considered in this paper is shown schematically in figure 1 . It consists of a copper cylindrical hollow cathode with an inner radius of a few millimeters and a length of a few centimeters, bounded on either side by a thin dielectric ring and an anode ring of the same inner radius. The geometry is symmetric around the center of the cathode (both axially and azimuthally). A DC voltage of a few hundred volts is applied between the cathode and anodes to sustain the discharge. The discharge gas is typically helium with a small admixture of argon at an intermediate gas pressure of a few $\mathrm{kPa}$, with argon ions dominating the discharge. Some typical parameter values are given in Table 1; these values will be used to evaluate the analytical expressions derived in this paper.

The discharge operation is illustrated in Figure 2 by the results of our previous twodimensional numerical simulations [16-18]. These simulations are based on the self-consistent solution of continuity and drift-diffusion equations for different plasma particle species, an electron energy equation, and Poisson's equation. The particle species taken into account are electrons and different ionic and excited neutral species of helium, argon, and copper. Particle source terms due to ionization, excitation, and other plasma chemistry are calculated using rate coefficients as a function of the electron mean energy. Wall recombination, secondary electron emission, and copper sputtering are accounted for by wall-flux boundary conditions. 
Figure 2 shows that, owing to the presence of a plasma, the anode potential propagates along the cylinder axis inside the cathode, such that most of the applied voltage falls in the radial direction across a thin space-charge sheath in front of the cathode, called cathode fall region or simply cathode fall (CF) in this paper. Outside the CF, the potential is close to the anode potential, with small radial variations of the order of the electron temperature to ensure quasineutrality of the plasma, and a relatively small axial gradient to ensure continuity of the plasma current.

The electron and ion transport in the $\mathrm{CF}$ are mainly radial and control the current and plasma density. The plasma is sustained by volume ionization (figure $2 \mathrm{~d}$ ) using the energy absorbed by electron acceleration in the $\mathrm{CF}$ (figure 2e), similar to the plasma in the negative glow region of conventional DC discharges, and is therefore regarded entirely as a negative glow plasma. What distinguishes this discharge from conventional DC discharges is that the ions created in the plasma are all transported to the cathode and make an important contribution to the secondary electron emission, so that less electron multiplication in the $\mathrm{CF}$ is needed to sustain the discharge. As a result, the CF becomes thinner, the ion density in the CF higher, and the discharge operates at higher current density. We will analyze this in the first part of this paper, in sections 3-4.

The discharge develops non-uniformity along the axial direction in order to generate an axial electron current that transports all created electrons to the anode. This is analyzed in the next part of this paper, in sections 5-6. We will show that the non-uniformity involves an axial electric field from which the plasma electrons absorb additional energy, leading to a shift in the current-voltage characteristic as a function of cathode length.

The potential profile inside the hollow cathode (figure 2a) traps the electrons in the radial direction, which makes it possible to sustain the discharge at low gas pressures where the electron mean free path exceeds the plasma size. The electrons accelerated in the CF then make multiple passes through the plasma at high velocity, each time reflected by the CF on the other side. This phenomenon is known as the pendulum effect and is generally considered essential for the HCD operation. The pendulum effect cannot be described by electron fluid equations and was studied previously by electron particle simulations [4,7] and a nonlocal approximation of the electron Boltzmann equation [6]. However, our previous simulations (figure 2) are based on electron fluid equations and thus neglect the pendulum effect. To get 
more insight in the role of the pendulum effect and the possible consequences of neglecting it (in our fluid simulations), we consider in this paper the following two limit cases. First, we will derive the analytical model (sections 3-6) by assuming that the electron mean free path is much shorter than the plasma radius, so that the pendulum effect can be neglected, as in the fluid simulations. Then, in sections 7-8, we will revisit the equations derived in the previous sections, assuming that the electron mean free path is very much larger than the plasma radius. The reality can be expected to be in between these two cases. We will show that some of the main principles of the HCD operation are similar for the two cases.

\section{Self-sustainment condition}

It is well known that in DC discharges, the thickness of the CF adjusts to satisfy the selfsustainment condition: an electron emitted from the cathode by ion impact must cause, during its life time, the creation of enough ions to ensure the emission of a new electron. More precisely, if $\gamma$ is the secondary emission coefficient characterizing the effective emission probability per ion, then each emitted electron must create exactly $1 / \gamma$ ions that are transported to the cathode. In conventional DC discharges, most of the ions impacting the cathode are created in or near the CF. The emitted electrons must then multiply by a factor $1+1 / \gamma$ as they cross the $\mathrm{CF}$ region. Equating this factor to $\exp (\alpha d)$, the Townsend expression for the electron multiplication factor, we obtain the well known self-sustainment condition [19]

$$
d \approx \frac{1}{\alpha} \ln \left(1+\frac{1}{\gamma}\right),
$$

where $d$ is the CF thickness and $\alpha$ is the Townsend coefficient, characterizing the mean ionization probability per unit traveled length, which is relatively constant for the high electron energies in the CF. In the steady state of conventional DC discharges, the CF has developed such that its thickness approximately satisfies equation (1).

However, equation (1) does not hold for HCDs because the ions created in the plasma beyond the CF are nearly all transported to the cathode and make an important contribution to the secondary electron emission, almost regardless of their position of creation. Let us analyze this for the HCD geometry of figure 1. The electron and ion transport across the CF is essentially radial. We assume that the CF thickness $d \ll<$ cathode inner radius $R$ so that the 
curvature of the $\mathrm{CF}$ can be neglected, i.e. the radial position coordinate $r$ behaves as a Cartesian coordinate for $R-d<r<R$. For simplicity, we also assume that the coefficients $\alpha$ and $\gamma$ are constant and that the electrons have so many collisions that they quickly lose their high energy after leaving the $\mathrm{CF}$, so that they cannot penetrate in the $\mathrm{CF}$ on the other side of the plasma (i.e. we neglect the pendulum effect).

According to the Townsend model [19], the electron flux $\Gamma_{\mathrm{e}}$ in the CF grows exponentially as a function of distance from the cathode:

$$
\Gamma_{e}(r)=-\gamma \Gamma_{i}(R) \exp (\alpha(R-r))
$$

the ion flux $\Gamma_{\mathrm{i}}$ follows from current conservation:

$$
\Gamma_{i}(r)=\frac{J}{e}\left(1-\frac{\gamma}{1+\gamma} \exp (\alpha(R-r))\right)
$$

where $J=e\left(\Gamma_{i}-\Gamma_{e}\right)$ is the current density which is constant across the $\mathrm{CF}$ (for $\mathrm{d} \ll<\mathrm{R}$ ). From equation (3) we can observe that if a significant ion flux enters the CF from the plasma, $\Gamma_{i}(R-d)>0$, then the CF must be thinner than in equation (1):

$$
d<\frac{1}{\alpha} \ln \left(1+\frac{1}{\gamma}\right)
$$

However, to find the value of the CF thickness $d$ we need to know how many ions are created in the plasma beyond the CF. This can be estimated from the electron energy balance, e.g. by balancing the average energy absorption and the energy losses associated with the creation and loss of a single electron-ion pair, as follows. We assume that the radial profile of the potential $\Phi$ in the CF is parabolic so the electric field $E$ is linear:

$$
\begin{gathered}
\Phi(r)=-V(1-(R-r) / d)^{2} \\
E(r)=-\frac{\partial \Phi}{\partial r}=-\frac{2 V}{d}(1-(r-R) / d)
\end{gathered}
$$

where $V$ is the total voltage. Combining equations (2) and (6), the electric energy absorbed by electrons in the $\mathrm{CF}$ is then

$$
H_{\mathrm{CF}}=-\frac{1}{\Gamma_{i}(R)} \int_{R-d}^{R} e \Gamma_{e}(r) E(r) d r=2 \frac{\exp (\alpha d)-1-\alpha d}{(\alpha d)^{2}} \gamma e V,
$$

on average per ion impacting the cathode. Since each electron-ion pair created in the volume corresponds to an ion impacting the cathode, $\mathrm{H}_{\mathrm{CF}}$ from equation (7) is directly the average absorbed energy per electron-ion pair. This absorbed energy depends on the electron 
multiplication because also the new electrons created in the CF absorb energy, leading to 'multiplication' of the absorbed energy. So the thicker the CF, the higher the absorbed energy per electron-ion pair. The lower limit for the absorbed energy is $\gamma e V$, corresponding to the case $d=0$ when the emitted secondary electrons do not multiply in the CF.

The electron energy losses can be conveniently represented by the energy per electron-ion pair $\mathrm{W}$, which is a rather constant parameter, typically a few times the ionization potential, determined mainly by the discharge gas and weakly dependent on the electron energy (for the energy range of interest here). This parameter $\mathrm{W}$ is often used and given in the literature for high-energy electron beams [20] but it can be defined for gas discharges in general [21,22]. For our HCD, the total energy per electron-ion pair consists of different contributions due to collisions and transport [21]:

$$
W=\frac{\text { lost power }}{\text { ionization rate }} \frac{\sum_{j=\text { collision }} \varepsilon_{j} \chi_{j} k_{j}}{\sum_{i=\text { ionization }} \chi_{i} k_{i}}+W_{\text {tr }}
$$

where $\varepsilon$ is the mean energy lost in a collision (constant for inelastic collisions), $k$ is the rate coefficient, $\chi$ is the fractional density of the target species, and the sums run over all collision processes $j$ and over the ionization processes $i$ only. The last term $W_{\text {tr }}$ is the energy loss associated with electron transport loss to the anode, which is effectively felt in the cathode region by thermal conductivity. From the numerical simulation results, the two terms of equation (8) can be estimated to be approximately $37 \mathrm{eV}$ and $13 \mathrm{eV}$, adding up to $\mathrm{W} \approx 50 \mathrm{eV}$ (see table 1). For simplicity we assume that $\mathrm{W}$ is constant.

Equating the absorbed energy $\mathrm{H}_{\mathrm{CF}}$ and the lost energy $\mathrm{W}$, we find:

$$
2 \frac{\exp (\alpha d)-1-\alpha d}{(\alpha d)^{2}} \gamma e V=W \text {. }
$$

This energy balance can be seen as a self-sustainment condition for HCDs and controls the CF thickness $d$, meaning that $d$ adjusts such that the right amount of energy is absorbed to create the ions necessary to sustain the discharge by secondary emission. The left-hand side of equation (9) is a monotonically rising function of $\alpha d$ that can be well approximated by Taylor expansion (of its logarithm) so that $d$ can be solved. This yields:

$$
d \approx \frac{6}{\alpha}\left(\sqrt{1-\ln \left(V / V_{\max }\right)}-1\right) \approx \frac{3}{\alpha}\left(\frac{V_{\max }}{V}-1\right)
$$


where $V$ cannot exceed the maximum voltage

$$
V_{\max }=\frac{W}{e \gamma} .
$$

The first approximation in equation (10) is accurate to within a few percent over the entire range of $V$. The second simpler approximation is valid only in case the CF is so thin that $\alpha d<$ 0.3 but in practice this is often the case; this expression was given previously in the context of HCDs [9]. The exact solution of equation (9) and the approximate solutions (10) are shown in figure 3. The maximum voltage (11) corresponds to the point where the energy balance is satisfied without electron multiplication in the CF. Beyond this voltage the absorbed energy always exceeds the energy loss, regardless of $d$, so the energy balance cannot be satisfied. Substituting some typical values $\gamma=0.1$ and $\mathrm{W}=50 \mathrm{eV}$ from table 1 , we find a maximum voltage of $500 \mathrm{~V}$.

\section{Radial ion transport}

Given the CF thickness, the ion density $n_{i}$ in the CF follows from Poisson's equation:

$$
e n_{\mathrm{i}}=-\varepsilon_{0} \frac{\partial^{2} \Phi}{\partial r^{2}}=\varepsilon_{0} \frac{2 V}{d^{2}},
$$

where we have used equation (5) and neglected the electron charges and the curvature of the $\mathrm{CF}$ (as before). From this, the current density can be estimated as

$$
J=(1+\gamma) e \Gamma_{i}(R) \quad(1+\gamma) e n_{\mathrm{i}} u_{i},
$$

where $\mathrm{u}_{\mathrm{i}}$ is the ion mean velocity in the center of the CF fall. Note that the ion velocity can be expected to vary across the $\mathrm{CF}$ much more than the flux $\Gamma_{\mathrm{i}}$, which is not fully consistent with our assumption of uniform $\mathrm{n}_{\mathrm{i}}$ and parabolic $\Phi(r)$, but the resulting errors are minimized if we take $u_{i}$ in the center of the $C F$ at position $r=R-d / 2$. Assuming that $u_{i}$ is of the form $\beta E^{1 / 2}$, which a good approximation for collisional noble gas ions in high field [19], the current density is then

$$
J \quad(1+\gamma) e n_{\mathrm{i}} \beta \sqrt{\frac{V}{d}}=\frac{2(1+\gamma) \varepsilon_{0} \beta V^{3 / 2}}{d^{5 / 2}} .
$$

On the other hand, if the ion mean free path $>d$ so that $\mathrm{u}_{\mathrm{i}}$ is limited by inertia rather than collisions, the current density is 


$$
J \quad(1+\gamma) e n_{\mathrm{i}} \sqrt{\frac{e V}{2 m_{i}}}=\frac{2^{1 / 2}(1+\gamma) e^{1 / 2} \varepsilon_{0} V^{3 / 2}}{m_{i}^{1 / 2} d^{2}},
$$

where $m_{i}$ is the ion mass. This latter situation can arise in gas mixtures where the dominant ions correspond to a minority component of the mixture so that they undergo few charge transfer collisions, e.g. $\mathrm{Ar}^{+}$ions in He-Ar mixtures with a small Ar fraction.

When substituting equation (10) in equation (14/15), as in figure 4, we see that the current density $J$ increases rapidly as $V$ approaches the maximum voltage $V_{\max }$ and the CF thickness approaches zero. This an important advantage of HCDs, essential for the hollow cathode effect: the current density can become much higher than in standard DC discharges because the self-sustainment condition allows a very thin CF. In fact, HCDs are usually operated at high current density close to the maximum voltage $V \approx V_{\max }$ to within $10 \%$ or so. This implies that $\alpha d<0.3$ and that most ionization happens in the plasma rather than the $\mathrm{CF}$, so that the ion flux is very nearly constant across the $\mathrm{CF}$ :

$$
\Gamma_{i}(R-d) \approx \Gamma_{i}(R)=\frac{J}{(1+\gamma) e}
$$

to within a few $\%$ as can be seen from equation (3). In the following we neglect that some ions are created in the $\mathrm{CF}$ and assume that the ion flux is totally created in the plasma; we will simply write $\Gamma_{\mathrm{i}}$ for the ion flux anywhere in the $\mathrm{CF}$.

Given the ion creation in the plasma, the plasma density $n$ is determined by the ion transport losses to the CF. Following the standard elementary theory for DC positive columns and many other discharges $[19,22]$, we assume that the electrons in the plasma have a uniform temperature $T_{e}$ that controls both their pressure and ionization frequency $v_{\mathrm{iz}}$. The ions are transported by the ambipolar electric field $E_{\mathrm{amb}} \approx-T_{e} \nabla n / n$ resulting from the electron momentum balance. Neglecting axial ion transport, the ion transport equation is

$$
\frac{1}{r} \frac{\partial}{\partial r}\left(r \mu_{i} E_{\mathrm{amb}} n(r)\right)=-\mu_{i} T_{e} \frac{1}{r} \frac{\partial}{\partial r}\left(r \frac{\partial n}{\partial r}\right)=v_{\mathrm{iz}}\left(T_{e}\right) n(r)
$$

where $\mu_{\mathrm{i}}$ is the ion mobility at low electric field. The solution is a Bessel profile:

$$
n(r)=n_{0} \tilde{J}_{0}\left(k_{r} r\right) \quad k_{r}=\sqrt{\frac{v_{\mathrm{iz}}}{\mu_{i} T_{e}}} .
$$


Neglecting the CF thickness $(d<<R)$ and the ion density in the CF $\left(n_{i}<<n_{0}\right)$, we impose the boundary condition that the ion density is zero at the cathode, which yields an implicit equation for the electron temperature:

$$
\frac{v_{\mathrm{iz}}\left(T_{e}\right)}{T_{e}}=\mu_{i}\left(\frac{2.405}{R}\right)^{2}
$$

Since $v_{\mathrm{iz}}\left(T_{e}\right)$ is a very steep function, $T_{e}$ is determined quite precisely by this equation and not very sensitive to changes in the gas density or radius $R$. The amplitude of the plasma density profile can be found by equating the total ionization rate (integrated over the cross section of the plasma) to the ion flux in the CF (integrated over its circumference):

$$
v_{\mathrm{iz}} 2 \pi \int_{0}^{R} n(r) r d r=v_{\mathrm{iz}} \pi R^{2} \bar{n}=2 \pi R \Gamma_{i},
$$

hence the radial-average plasma density is

$$
\bar{n}=\frac{2}{R v_{\mathrm{iz}}} \Gamma_{i}=\frac{2 R}{2.405^{2}(1+\gamma) e \mu_{i} T_{e}} J .
$$

i.e. the plasma density is proportional to the current density in the CF. Equation (21) can be combined with equation (14/15) to find the plasma density from the CF voltage.

\section{Axial electron transport}

While the ions are lost by radial transport to the cathode, the electrons are lost by transport in the axial direction toward the anode. This requires an axial electric field $E_{z}$ in the plasma, corresponding to a decrease of the $\mathrm{CF}$ voltage $\mathrm{V}$ as a function of the axial distance $z$ from the anode, and leads to a decrease of the plasma density $\bar{n}$ and the CF current density $J$ as a function of $z$. In this section we derive the axial profiles $J(z), \bar{n}(z), V(z)$, etc, from the equations for the axial electron transport. The axial coordinate $z$ is defined in figure $1 \mathrm{~b}$.

The axial electron flux results from both the electric drift and diffusion due to the density gradient. Integrated over the cross section of the plasma, the electron continuity equation with drift-diffusion flux is

$$
\pi R^{2} \frac{\partial}{\partial z}\left(-\mu_{e} E_{z} \bar{n}-\mu_{e} T_{e} \frac{\partial \bar{n}}{\partial z}\right)=2 \pi R(1+\gamma) \Gamma_{i}
$$


where $\mu_{e}$ is the electron mobility and the right-hand side is the total rate of electron creation by secondary emission and volume ionization. We assume that $\mu_{e}$ and $T_{e}$ are independent of $z$ and substitute equations (21) and (16):

$$
\frac{\partial}{\partial z}\left(\frac{E_{z}}{T_{e}} J+\frac{\partial J}{\partial z}\right)=-(1+\gamma)\left(\frac{2.405}{R}\right)^{2} \frac{\mu_{i}}{\mu_{e}} J,
$$

where we have chosen to work with the CF current density J; however, this is directly proportional to the average plasma density $\bar{n}$. To calculate the axial profiles, we could now substitute $E_{z}=-\partial V / \partial z$ and $J(V)$ from equation (14/15) and then try to solve for $V(z)$, but this is cumbersome. Rather, we linearize equation (23) by assuming that $E_{z}$ is uniform, in agreement with the numerical simulation results in figure 2 . As a first boundary condition we assume that $J=0$ at the cathode-anode interface at $z=0$. This is consistent with the fact that the plasma density drops to zero within a small distance $\sim \mathrm{R}$ inside the anode, as is shown by the numerical results in figure 2. For the second boundary condition, considering the axial symmetry of the geometry, we impose that the axial electron flux is zero at the position $z=L$ corresponding to the center of the cathode:

$$
\frac{E_{z}}{T_{e}} J(L)+\left[\frac{\partial J}{\partial z}\right]_{z=L}=0
$$

where the two terms come from drift and diffusion. (Note that, taking $\mathrm{E}_{\mathrm{z}}$ constant, we cannot impose the Neumann boundary condition $\partial \mathrm{J} / \partial \mathbf{z}=0$ because this would lead to nonphysical inflow of electrons at $\mathrm{z}=\mathrm{L}$.)

The appropriate solution of equation (23) is

$$
J(z)=J_{0} \exp \left(-k_{1} z\right) \sin \left(k_{2} z\right)
$$

where $\mathrm{J}_{0}$ is a normalization constant and $\mathrm{k}_{1}$ and $\mathrm{k}_{2}$ are space constants given by

$$
\begin{gathered}
k_{1}=\frac{E_{z}}{2 T_{e}} \\
k_{2}=\sqrt{k_{0}^{2}-k_{1}^{2}} \\
k_{0}=\sqrt{1+\gamma} \frac{2.405}{R} \sqrt{\frac{\mu_{i}}{\mu_{e}}} .
\end{gathered}
$$

In these equations, the axial electric field $E_{z}$ is not a free input parameter, but an eigenvalue parameter (similar to $T_{e}$ in equations (18-19)) to be determined from the boundary condition 
(24). Together with (27), this boundary condition yields the following implicit equations for $k_{1}$ and $k_{2}$ :

$$
\frac{\sin \left(k_{2} L\right)}{k_{2} L}=\frac{1}{k_{0} L} \quad k_{1}=-k_{0} \cos \left(k_{2} L\right),
$$

whose solution is shown in figure 5. In a good approximation (obtained by Taylor expansion around $\mathrm{k}_{2} \mathrm{~L}=\pi$ ) equation (29) yields

$$
k_{1} \approx k_{0}\left(1-\frac{\pi^{2}}{8}\left(\sqrt{\frac{4}{k_{0} L}+1}-1\right)^{2}\right) .
$$

This determines the axial electric field through equation (25):

$$
E_{z}=2 T_{e} k_{1}
$$

i.e. the field adjusts to satisfy the boundary conditions. Note that the value of $E_{z}$ selected by our boundary conditions is also the smallest $E_{z}$ value allowed by the general solution of equation (23), corresponding to the most uniform current density $J(z)$.

Equations (30-31) show that the axial electric field depends on the cathode length. The field is weaker as the cathode is shorter, decreasing from $2 T_{e} k_{0}$ for very long cathodes, to zero for $k_{0} L$ $=\pi / 2$. For still shorter cathodes the electric field is reversed:

$$
L<\frac{\pi}{2 k_{0}} \Rightarrow E_{z}<0
$$

Substituting the values from Table 1 , we find that $\mathrm{k}_{0}=201 \mathrm{~m}^{-1}$ so the field reversion occurs for $\mathrm{L}<7.8 \mathrm{~mm}$, which agrees quite well with our previous numerical simulation results $[17,18]$ (note that in these references, the cathode length $l$ corresponds to the whole cathode cavity, i.e. $l=2 L$ ). Figure 6 shows a comparison, for different cathode lengths, of the analytical potential $-\mathrm{E}_{\mathrm{z}} \mathrm{z}$ and the on-axis potential in the numerical simulations. This figure also compares the analytical current density profile (25) with the current density at the cathode in the numerical simulations. Note that for very short cathodes with strong field reversion $(\mathrm{L}=5 \mathrm{~mm})$ the assumption of constant $\mathrm{E}_{\mathrm{z}}$ is no longer reasonable and the analytical solution (25) is no longer appropriate. 


\section{Influence of axial electric field on self-sustainment}

To normalize the current density profile $\mathrm{J}(z)$ and calculate the total discharge current, we use equation (14/15) obtained from Poisson's equation, in combination with the CF thickness d from the energy balance. In order to do this rigorously, we should establish the energy balance integrated (or averaged) over the axial position z, since the energy is not necessarily lost at the same position where it has been absorbed, but nearly the same results can be obtained in a much simpler way by applying the energy balance locally at the position $\mathrm{z}=\mathrm{z}_{\mathrm{m}}$ where $\mathrm{J}$ is maximum. This maximum takes place some distance inside the cathode (for long enough cathodes with $\mathrm{k}_{0} \mathrm{~L}>\pi / 2$ ) and is given by

$$
\begin{gathered}
z_{\mathrm{m}}=\frac{\pi}{k_{2}}-L \\
J_{\mathrm{m}} \equiv J\left(z_{\mathrm{m}}\right)=J_{0} \frac{k_{2}}{k_{0}} \exp \left(-k_{1} z_{\mathrm{m}}\right),
\end{gathered}
$$

so the current density profile (25) can be written as

$$
J(z)=J_{\mathrm{m}} \frac{k_{0}}{k_{2}} \exp \left(-k_{1}\left(z-z_{\mathrm{m}}\right)\right) \sin \left(k_{2} z\right) .
$$

We now estimate $J_{m}$ by substitution of equation (10) in equation (14/15), as shown in figure 4 . The CF voltage $\mathrm{V}$ at $\mathrm{z}=\mathrm{z}_{\mathrm{m}}$ differs from the applied discharge voltage by the anode sheath voltage and by the axial voltage drop in the plasma $\mathrm{E}_{\mathrm{z}} \mathrm{z}_{\mathrm{m}}<2 \mathrm{~T}_{\mathrm{e}}$ but in total this voltage difference so small that it can be reasonably neglected. We simply use the applied voltage for $\mathrm{V}$ in equation (10).

However, the energy balance from section 3 requires an important modification in order to account for the axial electric field $E_{z}$. The electrons can absorb energy from this field, in addition to the energy $\mathrm{H}_{\mathrm{CF}}$ absorbed in the $\mathrm{CF}$. Although the axial field is relatively weak, it can have a significant influence because it acts upon all electrons everywhere in the plasma, whereas the CF field acts only upon part of the electrons in a small part of the volume. Per electron-ion pair, the energy absorbed from the axial electric field is

$$
H_{z}=-\frac{\pi R^{2}}{2 \pi R \Gamma_{i}}\left(-\mu_{e} E_{z} \bar{n}-\mu_{e} T_{e} \frac{\partial \bar{n}}{\partial z}\right) E_{z}
$$




$$
=(1+\gamma) 4 e T_{e} \frac{k_{1}^{2}}{k_{0}^{2}}\left(1+\frac{1}{2 k_{1} J} \frac{\partial J}{\partial z}\right) .
$$

The last factor in brackets, accounting for electron diffusion, is negligible near the point $z=z_{\mathrm{m}}$ as well as on average over the cathode length. Including the additional absorbed energy in the energy balance, $\mathrm{H}_{\mathrm{CF}}+\mathrm{H}_{\mathrm{z}}=\mathrm{W}$, we see that $\mathrm{H}_{\mathrm{z}}$ compensates for some of the energy losses, thereby reducing the maximum voltage in equation (10) and further. For the point $\mathrm{z}=\mathrm{z}_{\mathrm{m}}$ we find

$$
V_{\max }=\frac{W-H_{z}}{e \gamma}=\frac{1}{e \gamma}\left(W-(1+\gamma) 4 \frac{k_{1}^{2}}{k_{0}^{2}} e T_{e}\right)
$$

rather than equation (11). Upon substitution of the ratio $\mathrm{k}_{1} / \mathrm{k}_{0}$ from equation (30) and figure 5 , it turns out that the maximum voltage now depends on the cathode length, decreasing monotonically by approximately $4 T_{e} / \gamma$ as the cathode length is increased from very short to infinity.

The total discharge current $I$ is directly related to $\mathrm{J}_{\mathrm{m}}$ as

$$
I=2 \pi R \int_{0}^{L} J(z) d z=2 \pi R L \frac{\exp \left(k_{1} z_{\mathrm{m}}\right)}{k_{0} L} J_{\mathrm{m}} .
$$

Calculating this for different voltages $V$, we find the voltage-current characteristic $\mathrm{V}(\mathrm{I})$, which is shown in figure 7 for different cathode lengths and compared with numerical simulation results. The V-I curves for different cathode lengths are shifted in voltage due to different energy absorption $\mathrm{H}_{\mathrm{z}}$ from the axial field, following the trend of the maximum voltage (37). The shifts between the analytical curves are somewhat larger than between the numerical curves, which is consistent with the axial potential profiles in figure 6 , varying more in the analytical model than in the numerical simulations. Similar voltage shifts are observed in experimental V-I curves [17,18].

It is interesting to remark that most of the results in this and the previous section actually do not depend directly on the cathode length $L$, but rather on the parameter

$$
k_{0} L=2.405 \sqrt{1+\gamma} \sqrt{\frac{\mu_{i}}{\mu_{e}}} \frac{L}{R},
$$

i.e. on the aspect ratio length/radius, so decreasing the radius has the same effect as increasing the length. This was found earlier in [18]. The appearance of $k_{0} L$ as a scaling parameter is due to the fact that the characteristic time for transport losses is the same for the ions (lost by 
radial transport) as for the electrons (lost by axial transport), which is necessary to obtain a quasi-neutral plasma.

\section{Influence of pendulum effect on self-sustainment}

In the previous sections we assumed that the electrons have so many collisions that they rapidly lose their energy in the plasma and pass through the CF only once. This assumption is consistent with our previous fluid simulations, but its validity is doubtful. In fact, the electrons accelerated in the CF can have such a long mean free path that they penetrate in the $\mathrm{CF}$ on the other side of the plasma, and this is generally considered essential for the HCD operation. Therefore, in this section, we investigate the possible consequences of a long electron mean free path for the above analytical model. We consider the extreme limit case that the electron mean free path $\gg$ cathode radius. Then, the electrons coming from the $\mathrm{CF}$ oscillate in the potential well formed by the CF surrounding the plasma, passing through the plasma at high velocity many times before loosing their energy, each time penetrating into the $\mathrm{CF}$ on the other side and reflected by it. We will call these electrons fast electrons; their oscillating motion is the pendulum effect. As in section 3, we describe the electron collisions by a constant Townsend coefficient $\alpha$ (ionization probability per unit traveled length) and constant energy loss per ionization $\mathrm{W}$. We assume that the fast electrons are only forwardscattered in the collisions, so that their velocities remain in the radial direction, and that any new electrons are created with zero initial energy.

Let us first analyze how the pendulum effect modifies the self-sustainment condition (9) derived in section 3. Consider a secondary electron emitted from the cathode, becoming a fast electron. During a single pass through the plasma (from the CF to the CF on the other side) the electron is expected to produce $\sim 2 \alpha R$ ionizations and lose an energy $\sim 2 \alpha R W$, where we neglect the CF thickness $d \ll R$. As the electron loses energy, it can penetrate less deeply into the $\mathrm{CF}$. After $\mathrm{m}$ passes through the plasma the electron has lost an energy $2 m \alpha R W$ and penetrates into the $\mathrm{CF}$ up to a position $r_{m}$ given by

$$
e \Phi\left(r_{m}\right)=-e V\left(1-\left(R-r_{m}\right) / d\right)^{2}=-(e V-2 m \alpha R W),
$$

where we have used the potential profile from equation (5). Hence the number of times that the electron passes at a given position $r$ in the $\mathrm{CF}$ is 


$$
2 m(r)=\frac{e V}{\alpha R W}\left(1-(1-(R-r) / d)^{2}\right)
$$

where the factor 2 takes into account that the electron passes twice through the CF (forth and back) for each pass through the plasma. The expected number of ionizations that this electron produces inside the $\mathrm{CF}$ is

$$
\int_{R-d}^{R} 2 m(r) \alpha d r=\frac{2 d}{3 R} \frac{e V}{W}
$$

The resulting new electrons are accelerated through part of the CF voltage and also become fast electrons. The total energy absorbed from the CF by the secondary electron plus the new fast electrons is

$$
U=e V-\int_{R-d}^{R} 2 m(r) e \Phi(r) \alpha d r=e V\left(1+\frac{2 d}{15 R} \frac{e V}{W}\right) .
$$

Note that the new fast electrons create other fast electrons, which then also absorb energy, etc, but this additional energy is proportional to higher powers of $d / R$ and can be neglected for the case $d / R<<1$ considered here. Dividing the absorbed energy $U$ by the energy per ionization $W$, we find the total number of ionizations $M$ in both the CF and the plasma, due to a single secondary electron emitted from the cathode:

$$
M=\frac{U}{W}=\frac{e V}{W}\left(1+\frac{2 d}{15 R} \frac{e V}{W}\right) .
$$

A similar expression was given by [9] as an upper limit of the multiplication factor (except that $2 / 15$ is replaced by $1 / 4$ ). This number is much larger than the number of fast electrons created in the $\mathrm{CF}$ from equation (42), so most electrons are electrons created outside the CF in the plasma, where they remain at relatively low energy and form the bulk of the plasma. However, in our longitudinal HCD geometry these bulk electrons can absorb energy from the axial electric field and cause additional ionization. Therefore, in order to find the selfsustainment condition and CF thickness, we write again the electron energy balance per electron-ion pair, including the energy $H_{z}$ absorbed from the axial field:

$$
H_{\mathrm{CF}}+H_{z}=\gamma U+H_{z}=W
$$

which yields

$$
d=R \frac{15 W}{2 e V}\left(\frac{V_{\max }}{V}-1\right) \quad V_{\max }=\frac{W-H_{z}}{e \gamma} .
$$

This agrees with equation (10) in section 3 , if we replace

$$
\alpha \rightarrow \frac{2 e V}{5 W R} .
$$


Substituting the values from table 1 , we see that $\alpha=1333 \mathrm{~m}^{-1}$ is to be replaced by a somewhat larger value $\approx 2000 \mathrm{~m}^{-1}$. This suggests that for these conditions, the pendulum effect is important but not completely dominant. It also suggests that our previous fluid simulations somewhat underestimate the electron multiplication.

Note that according to some previous papers [4,6], the essence of the pendulum effect is that it allows the fast electrons to create new electrons inside the $\mathrm{CF}$, which also become fast electrons, and so on, leading to an exponential increase of fast electrons and absorbed energy (per secondary electron). However, this mechanism is not specific for the pendulum effect but is also present if the electrons pass through the CF only once. This is the reason why equation (46) has same form as equation (10) in section 3. The pendulum effect just allows the electrons to ionize the gas at arbitrarily low pressure. The discharge then becomes independent of the Townsend coefficient $\alpha$, according to equation (47).

\section{Influence of pendulum effect on plasma properties}

Finally, let us briefly discuss the consequences of the pendulum effect for the plasma properties. We need to take into account that there are two groups of electrons with different kinetic behavior. The fast electrons are responsible for most of the ionization but do almost not contribute to the plasma density and ambipolar field, which are determined mainly by the bulk electrons. Assuming that the bulk electrons can be characterized by a uniform temperature $T_{e}$ and neglecting the fast-electron density, the ion transport equation is

$$
-\mu_{i} T_{e} \frac{1}{r} \frac{\partial}{\partial r}\left(r \frac{\partial n}{\partial r}\right)=S_{\text {fast }}(r)+v_{\mathrm{iz}}\left(T_{e}\right) n(r),
$$

where $S_{\text {fast }}$ is the ionization rate due to fast electrons and $n, T_{e}$, and $\nu_{\mathrm{iz}}$ represent only the bulk electrons. The solution of this equation is no Bessel profile as in section 4 because the fastelectron ionization $S_{\text {fast }}(r)$ is not proportional to bulk-electron density $n(r)$. In fact, in view of the essentially radial motion of the fast electrons, $S_{\text {fast }}(r)$ can be expected to be peaked around the axis due to focusing of the electron trajectories. We will not calculate the radial profiles; we simply integrate equation (48) over the cross section of the plasma:

$$
2 \pi R \Gamma_{i}=-2 \pi R \mu_{i} T_{e}\left[\frac{\partial n}{\partial r}\right]_{r=R}=2 \pi \int_{0}^{R} S_{\text {fast }}(r) r d r+\pi R^{2} v_{\mathrm{iz}} \bar{n}
$$


The density gradient at the boundary can be written as

$$
\left[\frac{\partial n}{\partial r}\right]_{r=R}=C \frac{\bar{n}}{R}
$$

where $\mathrm{C}$ is a numerical constant which is approximately 3 to 4 for any kind of physically reasonable profile $\mathrm{n}(\mathrm{r})$; for a Bessel profile $\mathrm{C}=2.405^{2} / 2=2.892$. Hence, the first two members of equation (49) yield

$$
\bar{n}=\frac{R \Gamma_{i}}{C \mu_{i} T_{e}}=\frac{R}{C(1+\gamma) e \mu_{i} T_{e}} J
$$

which remains close to equation (21) in section 4 even if $n(r)$ is not exactly a Bessel profile. Furthermore, the integral of $S_{\text {fast }}$ must be consistent with the number of fast-electron ionizations calculated in equation (44):

$$
2 \pi \int_{0}^{R} S_{\text {fast }}(r) r d r=2 \pi R \gamma \Gamma_{i} M=2 \pi R \Gamma_{i}\left(1-\frac{H_{z}}{W}\right),
$$

where we have also used equation (45). Substituting equations (51-52) in equation (49), we obtain an equation for the bulk-electron temperature:

$$
\frac{v_{\mathrm{iz}}\left(T_{e}\right)}{T_{e}}=\frac{2 C \mu_{i}}{R^{2}} \frac{H_{z}}{W} .
$$

Comparing this with equation (19) in section 4, we see that the right-hand side is smaller by approximately a factor $\mathrm{H}_{\mathrm{z}} / \mathrm{W}$, leading to a lower electron temperature, and consequently to a higher plasma density from equation (21/51). Expressing the absorbed energy $\mathrm{H}_{\mathrm{z}}$ in terms of the axial electric field $\mathrm{E}_{\mathrm{z}}$, as in equation (36), equation (53) can also be written as

$$
\nu_{\mathrm{iz}}\left(T_{e}\right) W \approx e \mu_{e} E_{z}^{2} .
$$

This clearly shows that the bulk-electron temperature is directly controlled by the local axial field, exactly as in a DC positive column, and contrary to the electron temperature in section 4 without the pendulum effect. What is more, a lower electron temperature can be expected to reduce the axial field and the absorbed energy. According to section 5, both $\mathrm{E}_{\mathrm{z}}$ and $\mathrm{H}_{\mathrm{z}}$ are proportional to $T_{e}$ so that a decrease of $T_{e}$ causes a proportional decrease of $H_{z}$, which reduces $\mathrm{T}_{\mathrm{e}}$ even further, and so on. Substituting equation (36) in equation (53), we get

$$
\frac{v_{\mathrm{iz}}\left(T_{e}\right)}{T_{e}^{2}} \quad 8 \mathrm{C}(1+\gamma)\left(\frac{k_{1}}{k_{0}}\right)^{2} \frac{\mu_{i}}{W R^{2}} .
$$

The $T_{e}$ from this equation can indeed be much lower than that from equation (19). However, this result depends strongly on our assumption that all bulk electrons are created with zero 
initial energy. A more quantitative estimation of the bulk-electron temperature would involve treating the energy transfer from the fast electrons to the bulk electrons.

\section{Conclusions}

The analytical model presented in this paper gives the following insights in the operation of longitudinal HCDs:

- Since the ions created in the plasma are all collected by the cathode and cause secondary electron emission, electron multiplication in the $\mathrm{CF}$ is not necessary to sustain the discharge, but rather to satisfy the energy balance per electron-ion pair: the absorbed energy increases as a function of the electron multiplication in the $\mathrm{CF}$. The $\mathrm{CF}$ thickness adjusts such that the right amount of energy is absorbed to create the ions necessary to sustain the discharge by secondary emission.

- There is a maximum CF voltage beyond which the absorbed energy always exceeds the lost energy so that the energy balance cannot be satisfied. When increasing the voltage up to this maximum voltage, the $\mathrm{CF}$ thickness decreases down to zero, in order to minimize the absorbed energy. According to Poisson's equation, the ion density and current density in the CF then increase to infinity (or at least to such high values that the present model is no longer reasonable). The typical operation of the longitudinal $\mathrm{HCD}$ is close to this maximum voltage, with a thin $\mathrm{CF}$ where almost no electron multiplication takes place.

- The longitudinal HCD develops axial non-uniformity and an axial electric field in order to evacuate the created electrons to the anode, such that the characteristic time for transport losses is the same for electrons as for ions. The axial electric field necessary to achieve this is stronger as the cathode is longer, or more precisely, as the ratio cathode length / cathode radius is larger. For very short cathodes the axial electric field is reversed to slow down electron diffusion losses to the anode. 
- Additional energy absorption from the axial electric field in the plasma bulk, similar to electron heating in DC positive columns, modifies the energy balance per electron-ion pair and hence modifies the $\mathrm{CF}$ thickness. This leads to a shift in the maximum voltage depending on cathode length, resulting in a voltage-shift of the voltage-current characteristic. This is also observed in experiments and in our previous numerical simulations.

- In principle, the above mechanisms are not directly affected by or dependent on the pendulum effect. The pendulum effect just allows the ionization to take place for arbitrarily long electron mean free paths, imposing an effective lower limit 2eV/5WR on the Townsend coefficient $\alpha$. However, the pendulum effect can also be expected to reduce the temperature of the electrons in the plasma bulk, thereby reducing both the axial and the radial electric field in the plasma, slowing down the radial ion transport, and consequently increasing the plasma density for a given current. Our previous numerical fluid simulations could be unrealistic on these points. We remark nevertheless that our simulation results were found in good overall agreement with experimental data. More work is need to clarify this issue, e.g. using electron MonteCarlo simulation, as in [11,12].

\section{Acknowledgements}

The authors thank L. C. Pitchford and J. P. Boeuf of the LAPLACE Toulouse, J. J. A. M. van der Mullen of the TUE Eindhoven, and M. Grozeva of the ISSP Sofia for useful discussions and remarks. This work was done as part of "Physical chemistry of plasma-surface interaction" (PSI) in the framework of the Belgian federal programme "Interuniversity Attraction Poles" (IAP). The authors acknowledge the support of the Dutch National Programme BSIK, in the ICT project BRICKS, theme MSV1 and the support of the National Science Fund of Bulgaria, project DO 02-274/2008. 
References

[1] P.F. Little and A.v. Engel, "The hollow cathode effect and the theory of glow discharges", Proc. Phys. Soc. London A 224, 209 (1954)

[2] B. E. Warner, K. B. Person, and G. J. Collins, J. Appl. Phys. 50, 5694 (1973)

[3] E. M. van Veldhuizen and F. J. de Hoog, J. Phys. D: Appl. Phys. 17, 953-968 (1984)

[4] J. P. Boeuf, "Self-consistent models of DC and transient glow discharges", in Physics and Applications of Pseudosparks (NATO ASI Series B 219) ed M.A. Gundersen and G. Schaefer, pp. 255-275 (1990)

[5] K.H. Schoenbach, L.L. Vahala, G.A. Gerdin, N. Homayoun, F. Loke, "The effect of pendel electrons on breakdown and sustainment of a hollow cathode discharge", in Physics and Applications of Pseudosparks (NATO ASI Series B 219) ed M.A. Gundersen and G. Schaefer, pp. 293-301 (1990)

[6] V.I. Kolobov and L.D. Tsendin, Plasma Sources Sci.Technol. 4, 551 (1995)

[7] Z. Donko, K. Rozsa and R.C. Tobin, "Monte-Carlo analysis of the electrons' motion in a segmented hollow cathode discharge", J. Phys. D:Appl. Phys. 29, 105 (1996)

[8] R.R. Arslanbekov, R.C. Tobin and A.A. Kudryavtsev, "Self-consistent model of high current density segmented hollow cathode discharges", J. Appl. Phys. 81 (2), 554 (1997)

[9] R.R. Arslanbekov, A.A. Kudryatsev and R.C. Tobin, "On the hollow-cathode effect: conventional and modified geometry”, Plasma Sources Sci. Technol. 7, 310 (1998)

[10] A.J. Lichtenberg and M.A. Lieberman, "Modelling a metal-vapor buffer-gas hollow cathode discharge”, J. Appl. Phys. 87 (10), 7191 (2000)

[11] A. Bogearts and M. Grozeva, Appl. Phys. B: Lasers and Optics 75, 731-738 (2002)

[12] A. Bogearts and R. Gijbels, J. Appl. Phys. 92(11), 6408 (2002)

[13] F. Sigeneger and R. Winkler, "Self-consistent analysis of a helium plasma in a cylindrical hollow cathode”, Plasma Chem. and Plasma Proc. 25 (2), 147 (2005)

[14] F. Sigeneger, Z. Donko and D. Loffhagen, "Boltzmann equation and particle-fluid hybrid modelling of a hollow cathode discharge”, J. Appl. Phys. 38, 161 (2007) 
[15] I. G. Ivanov, E. L. Latush, and M. F. Sem, Metal vapour ion lasers (Wiley \& Sons, Chichister UK, 1996)

[16] D. Mihailova, M. Grozeva, G.J.M. Hagelaar, J. van Dijk, W.J.M. Brok and J.J.A.M. van der Mullen, J. Phys. D: Appl. Phys. 41, 245202 (2008)

[17] D. Mihailova, J. van Dijk, M.Grozeva, G.J.M. Hagelaar and J.J.A.M. van der Mullen, J. Phys. D: Appl. Phys. 43, 145203 (2010)

[18] D. Mihailova, Sputtering hollow cathode discharges designed for laser applications; experiments and theory, Ph.D. thesis (Eindhoven University of Technology, Netherlands, 2010)

[19] Y. P. Raizer, Gas discharge physics (Springer-Verlag, Berlin Heidelberg, 1991)

[20] J. M. Valentine, "Energy per ion pair for electrons in gases and gas mixtures", Proc. R. Soc. Lond. A 211, 75-85 (1952)

[21] M. A. Lieberman and S. Ashida, "Global models of pulse-power-modulated highdensity, low-pressure discharges", Plasma Sources Sci. Technol. 5, 145-158 (1996)

[22] M. A. Lieberman and A. J. Lichtenberg, Principles of plasma discharges and materials processing, Second Edition (John Wiley \& Sons, New Jersey, 2005) 
Tables

Table 1. Parameters of the HCD configuration considered in this paper. The exact definition of the parameters is given further on in the text. The values in the lower part of the table are estimates based on our previous numerical simulations.

Parameter Symbol Value

Cathode radius

$R \quad 2 \mathrm{~mm}$

Cathode length

$2 L \quad 2-8 \mathrm{~cm}$

Applied voltage

$V \quad 350-500 \mathrm{~V}$

Discharge current

$I$

$0.2-2 \mathrm{~A}$

Gas composition $95 \% \mathrm{He}-5 \% \mathrm{Ar}$

Gas density $1.666 \times 10^{23} \mathrm{~m}^{-3}$

Gas temperature $1000 \mathrm{~K}$

Electron temperature (plasma)

$T_{e} \quad 3.67 \mathrm{eV}$

Electron mobility $\mu_{e} \quad 11.4 \mathrm{~m}^{2} \mathrm{~s}^{-1}$

Ion mobility (plasma) $\mu_{i} \quad 0.29 \mathrm{~m}^{2} \mathrm{~V}^{-1} \mathrm{~s}^{-1}$

Ion mobility coefficient (CF)

$\beta \quad 15 \mathrm{~m}^{3 / 2} \mathrm{~V}^{-1 / 2} \mathrm{~s}^{-1}$

Townsend coefficient

$\alpha \quad 1.3 \times 10^{3} \mathrm{~m}^{-1}$

Secondary emission coefficient

$\begin{array}{ll}\gamma & 0.1\end{array}$

Energy per electron-ion pair

W

$50 \mathrm{eV}$ 
Figure captions

Figure 1. Geometry of the longitudinal HCD: (a) schematic overview of the discharge tube containing a cathode cylinder (C) and two anode rings (A); (b) definition of the coordinate axes used in this paper.

Figure 2. Spatial profiles of the main plasma parameters as obtained in our previous numerical simulations. The simulation domain corresponds to figure $1 \mathrm{~b}$. The cylinder axis is in the bottom of each plot, the cathode center is on the right, and the anode and cathode surfaces are in the top as indicated in the first plot. The half cathode length $\mathrm{L}=2.5 \mathrm{~cm}$, the other discharge parameters are given in table 1.

Figure 3. Relation between the CF voltage $\mathrm{V}$ and the CF thickness $\mathrm{d}$ as given by the energy balance (9). Solid line: exact solution. Dotted line: first approximation in equation (10). Dashed line: second approximation in equation (10).

Figure 4. Current density at the cathode as a function of CF voltage, obtained by substitution of equation (10) in equation (14) (drift) and equation (15) (free fall), using the parameters in table 1 and the $\mathrm{Ar}^{+}$ion mass. Only the lowest curve is physically valid, equation (14) in most of the current density range.

Figure 5. Solution of equation (29). Solid line: exact solution. Dashed line: approximate solution (30).

Figure 6. Axial profiles of the CF current density and the on-axis potential from the analytical model equations $(25,30-31)$ and from the numerical simulations, for different half cathode lengths $\mathrm{L}$, indicated in $\mathrm{mm}$ with the curves.

Figure 7. Voltage - current characteristics for different half cathode lengths L from the analytical model, combining equations (14, 30, 37-38), compared with numerical simulations [18]. Except for the cathode length, the input parameters for the analytical curves are constant as given in table 1 . 
(a)
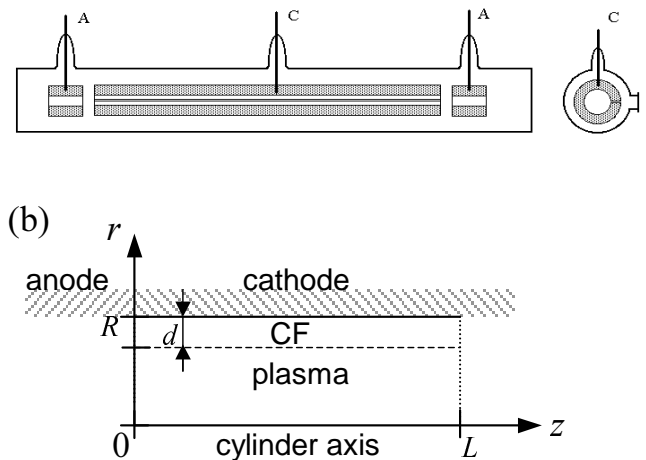

Figure 1 (fig1.eps) 
(a) Potential (contour interval $5 \mathrm{~V}$ )

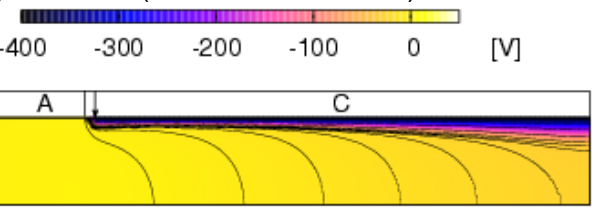

(c) Electron mean energy

$\begin{array}{lllllll}0 & 20 & 40 & 60 & 80 & 100 & {[\mathrm{eV}]}\end{array}$

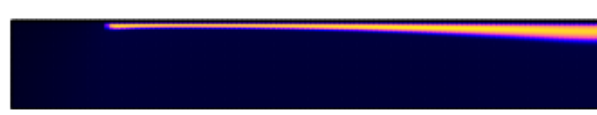

(e) Ionisation source term

$\begin{array}{llllll}0 & 1 & 2 & 3 & 4 & 5\end{array}\left[10^{25} \mathrm{~s}^{-1} \mathrm{~m}^{-3}\right]$

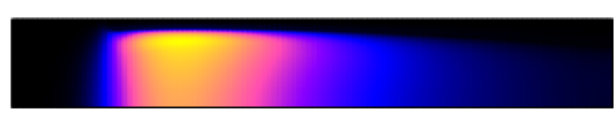

(b) Electron density

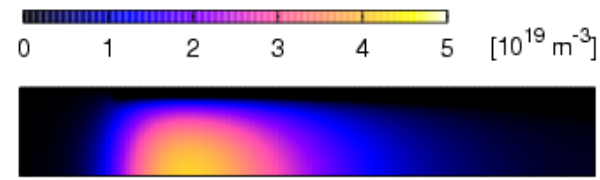

(d) Electron energy in plasma (contours $0.2 \mathrm{eV}$ )

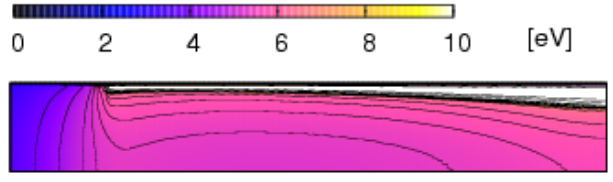

(f) Absorbed power density

Figure 2 (fig2.eps) 


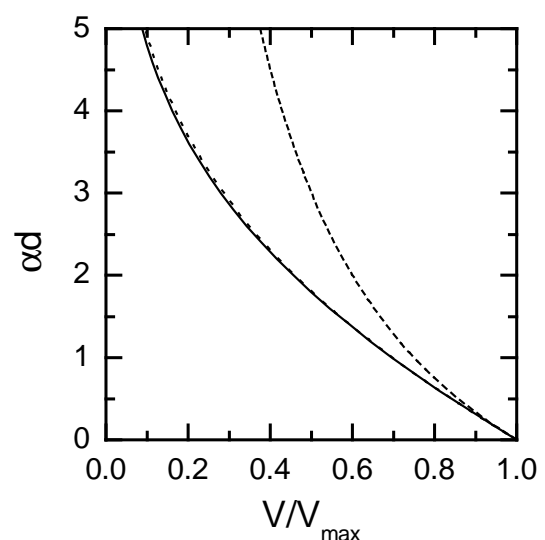

Figure 3 (fig3.eps) 


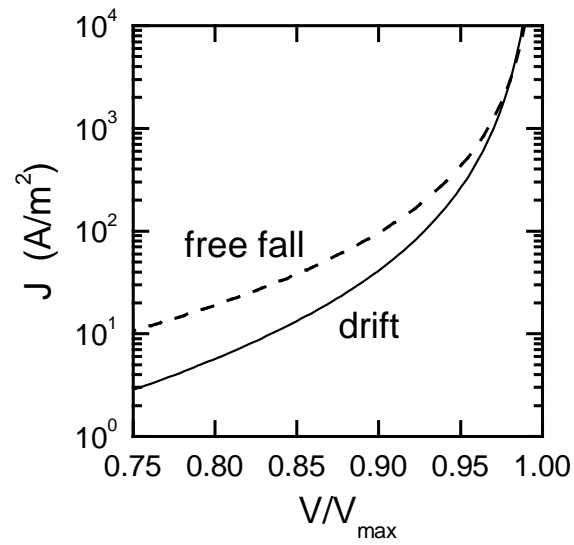

Figure 4 (fig4.eps) 


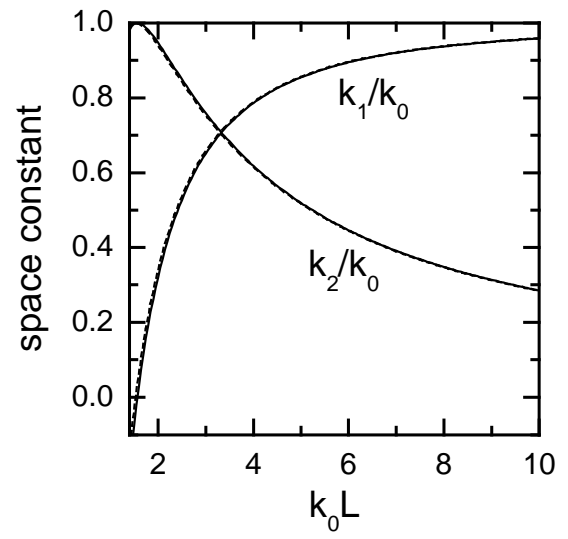

Figure 5 (fig5.eps) 

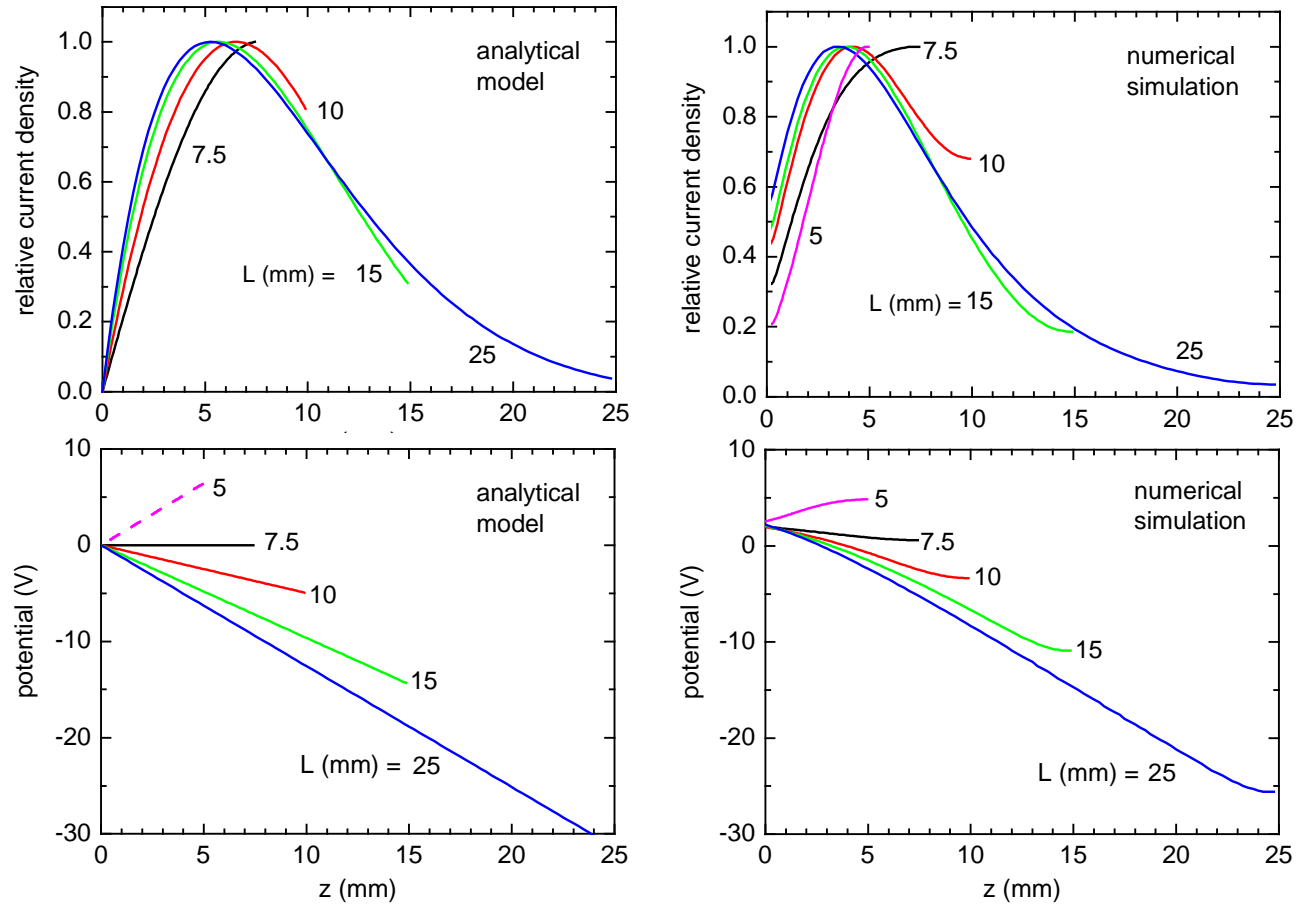

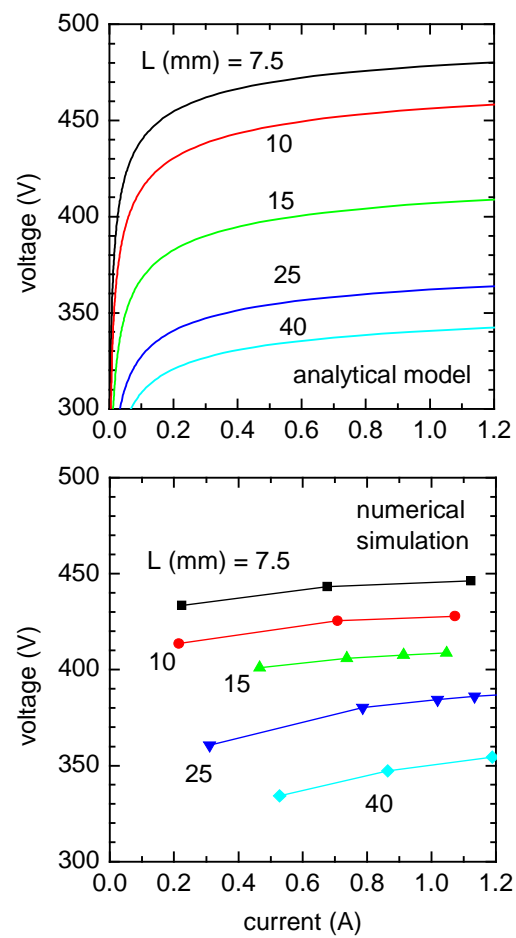

Figure 7 (fig7.eps) 